\title{
Nils-Olof Jönsson, Vie et miracles de saint Josse de Jean Miélot
}

\section{Maria Colombo Timelli}

\section{(2) OpenEdition}

10 Journals

\section{Édition électronique}

URL : http://journals.openedition.org/studifrancesi/29996

DOI : 10.4000/studifrancesi.29996

ISSN : 2421-5856

Éditeur

Rosenberg \& Sellier

\section{Édition imprimée}

Date de publication : 1 avril 2006

Pagination : 135-136

ISSN : 0039-2944

\section{Référence électronique}

Maria Colombo Timelli, « Nils-Olof Jönsson, Vie et miracles de saint Josse de Jean Miélot », Studi

Francesi [En ligne], 148 (XLX | I) | 2006, mis en ligne le 30 novembre 2015, consulté le 18 avril 2021.

URL : http://journals.openedition.org/studifrancesi/29996 ; DOI : https://doi.org/10.4000/

studifrancesi.29996

Ce document a été généré automatiquement le 18 avril 2021.

\section{(c)}

Studi Francesi è distribuita con Licenza Creative Commons Attribuzione - Non commerciale - Non opere derivate 4.0 Internazionale. 


\title{
Nils-Olof Jönsson, Vie et miracles de saint Josse de Jean Miélot
}

\author{
Maria Colombo Timelli
}

\section{RÉFÉRENCE}

NILS-OLOF JÖNSSON, Vie et miracles de saint Josse de JEAN MIÉLOT, Turnhout, Brepols, 2004

(«Textes vernaculaires du Moyen Age», 1), pp. 168.

1 Traducteur, copiste, peut-être chef d'un atelier à Lille, au service de Philippe le Bon, puis de Louis de Bruges, Jean Miélot est une figure qui mériterait certainement d'être mieux connue, au même titre qu'un David Aubert ou un Jean Wauquelin, objet -ces deux derniers- de monographies récentes (Richard Straub, David Aubert, 'escripvain' et 'clerc', Amsterdam-Atlanta, Rodopi, 1995: cf. SF, 124, 1998, p. 112) ou d'Actes de Colloque (Les manuscrits de David Aubert, 'escrip-vain' bourguignon, Textes réunis par Danielle Quéruel, Paris, Presses de l'Université de Paris-Sorbonne, 1999: cf. SF, 133, 2001, p. 124; Jehan Wauquelin. De Mons à la Cour de Bourgogne, sous la dir. de Marie-Claude de Crécy, Turnhout, Brepols, 2006), outre que d'éditions de textes. Ce livre de Nils-Olof Jönsson ne contribue que d'une manière très partielle à une meilleure connaissance de Miélot et de sa production; il s'agit ici de l'édition d'un manuscrit autographe (la cote apparaît sous deux formes dans l'introduction: Bruxelles, KBR, ms. 10958 et 10598, la première étant la bonne), dont est connue aussi une copie, conservée à la Bibliothèque Municipale de Valenciennes (et non pas Valence, comme on lit dans la note 61:18, p. 100), ms. 511. Le manuscrit bruxellois est un recueil de textes entièrement consacré à Saint Josse, à l'égard duquel Philippe le Bon avait une vénération particulière; il contient en effet: une vie du Saint en vers illustrée (47 miniatures accompagnées chacune d'un quatrain: 13 d'entre elles sont reproduites aux pp. 153-165. À ce propos, on aurait souhaité une analyse du rapport texte-image: N.-O. Jönsson se limite à rapporter le jugement de Frédéric Lyna sur les miniatures, "d'une qualité fruste et malhabile», p. IX, tout en ajoutant qu'elles "peuvent quelque peu nous aider à interpréter le texte»), une vie en prose, le récit des 37 miracles attribués au Saint. Des 
textes plus brefs complètent l'ensemble: une généalogie de Saint Josse, un épisode miraculeux (l'apparition de la main de Dieu pendant une messe), l'inventio du corps du Saint. Miélot est certainement l'auteur des traductions du latin: la vie en prose, l"apparition', l'invention («translatee -celle-ci- par le commandement et ordonnance» du Grand Duc), textes datés 1449 dans les explicit. Jönsson reconnaît la source du recueil dans un texte latin, la version amplifiée d'une Vita d'Isembardus dont ne sont conservés que trois manuscrits tardifs (deux du XVI ${ }^{\mathrm{e}}$, un du XVII ${ }^{\mathrm{e}}$ siècle).

2 L'introduction au texte n'est malheureusement pas généreuse en informations, ni pour ce qui concerne Jean Miélot (pour lequel on pourra se rapporter avec profit à la notice sur le ms. 9270 de la KBR de Bruxelles, dans La Librairie des ducs de Bourgogne, I, Turnhout, Brepols, 2000, pp. 114-115), ni sur le manuscrit de Bruxelles, dont le contenu n'est pas analysé; le rapport avec la probable source latine, dont manque encore une édition critique, est étudié de manière plus détaillée. Qui plus est, les aspects philologiques apparaissent négligés: on doit faire pleine confiance à l'éditeur pour ce qui est du caractère autographe du ms. $B$ ( $p$. VIII: «c'est un autographe de la main de Jean Miélot»), pour son appartenance à la bibliothèque de Philippe le Bon (pas de renvoi à Barrois, où le manuscrit porte les n.os 749 et 1972), ou encore pour la dérivation de $V$, le rapport entre les deux témoins étant donné pour acquis (p. X: «le ms. $n^{\circ} 511$ de la Bibliothèque municipale de Valenciennes est une copie du manuscrit écrit par Jean Miélot»). L'analyse linguistique mélange des constatations banales (par exemple, sur l'usage du partitif, p. XXV) et des relevés plus intéressants, peu exploités cependant (cf. les 25 néologismes, pour la plupart des emprunts au latin, dont la liste est donnée aux pp. XXX-XXXI: ces mots ne sont accompagnés d'aucun commentaire, ni des renvois au texte, ce qui aurait permis de les resituer rapidement dans leur contexte, sans passer par le glossaire). Certaines ingénuités sautent aux yeux; ainsi à propos de l'enchevêtrement de quelques phrases, juste avant la citation d'un fragment de Miélot particulièrement embrouillé, on lit: «A la place de la citation ci-dessous un auteur moderne aurait pu ordonner ses idées ainsi: ...» (suit la même phrase, 'traduite' en français moderne, p. XXVIII); de même, sur le procédé de la reduplicatio synonymica dans la traduction: "[à défaut d'édition du texte latin], il n'a pas été possible d'établir combien de réduplications sont dues au traducteur et combien sont simplement traduites du latin, mais je suis sûr que la majorité des réduplications ne se retrouvent pas dans le latin» (p. XXIX, c'est moi qui souligne).

3 Le texte semble édité avec soin, malgré deux pratiques bizarres: la numérotation de toutes les lignes (aussi bien les lignes imprimées que les lignes vides), l'indication du changement de feuillet à la fin des vers et non pas au début, ce qui confond relativement la lecture. On peut encore discuter certains choix, par exemple dans l'emploi des majuscules (nostre seigneur [= Dieu] -dans la plupart des cas avec initiales minusculesapparaît aussi sous la forme nostre Seigneur). Quelques corrections ou interventions de l'éditeur paraissent superflues (9,33: pluseus -qui reflète l'amuïssement de $r$ finalcorrigé en pluseurs; 29,18 et 37,23: tréma sur jeüne [= jeûne]; quelques interprétations sont à revoir (16,34-35: en toutes choses qu'il appartenoit et estoient de Dieu, construction asymétrique mais parfaitement acceptable en moyen français, est ainsi commentée en note, p. 88: “'qu'il était proche et qui étaient de Dieu'. Il semble que le sujet du deuxième verbe, qui, manque»; 55,3-4: «Et [deux demoniacles guéris] vesquirent depuis moult de temps en ce siecle songneux et diligens de raconter a tous peuples qui les vouloit ouir les bienfais que divinement ilz avoient receu»; l'incise et la valeur de qui 
n'étant pas reconnues, on lit dans la note, p. 98: «La forme du singulier du verbe doit être une faute d'attention du traducteur»). On relève d'autres affirmations naïves dans les notes: p. 93, 30:32 [à propos de la forme se envoleper] «métathèse de enveloper, non pas corrigée par le copiste du ms. V, comme [deux autres] métathèses»; p. 98, 51:15 «Le mot garde est féminin, mais Miélot utilise plusieurs fois le genre naturel [c'est moi qui souligne] du mot»; p. 100, 61:18 «Miélot utilise très rarement la lettre $k$... Il l'écrit avec difficulté; la lettre semble composée de $l$ et de $r$ ». De rares coquilles ne nuisent pas vraiment à la compréhension du texte (ainsi Lesqulles 44,14; mais le nom de LouisFernand Flutre est malencontreusement toujours graphié Fluthre, tant dans les notes que dans la Bibliographie).

4 Le texte est suivi d'une Table des noms propres (pp. 105-110), du Glossaire (pp. 111-142), qui enregistre les formes conjuguées / déclinées des mots variables, mais dont les critères d'inclusion des termes ne sont pas explicités, de la Bibliographie (pp. 143-150; quelques imprécisions: l'étude célèbre d'Alexandre Lorian concerne la prose narrative française du XVI [et non pas du XIV ${ }^{e}$ ] siècle (p. 147), le titre de la monographie de Richard Straub sur David Aubert est à corriger (Faux titre indiquant la collection de Rodopi et ne faisant pas partie de l'intitulé, p. 149; la Legenda aurea est désormais disponible dans l'édition critique de Giovanni Paolo Maggioni, Firenze, Le Lettere, 2000).

5 Si l'édition de Nils-Olof Jönsson a l'indiscutable mérite de mettre à la disposition des lecteurs un groupe de textes manifestement important dans la production du XV $\mathrm{XV}^{\mathrm{e}}$ siècle bourguignon, et ce tant pour l'hagiographie dans le milieu ducal que pour la production, encore peu et mal connue, de Jean Miélot, il nous semble que les apparats complémentaires sont à utiliser avec prudence. 